\title{
CARACTERIZAÇÃO DE MÃES E RECÉM-NASCIDOS PRÉ-TERMO EM UMA UNIDADE DE TERAPIA INTENSIVA NEONATAL
}

\section{CHARACTERIZATION OF MOTHERS AND PRETERM NEWBORNS IN A NEONATAL INTENSIVE CARE UNIT}

\section{CARACTERIZACIÓN DE MADRES Y RECIÉN NACIDOS PRETÉRMINOS EN UNA UNIDAD DE CUIDADO INTENSIVO NEONATAL}

Andréa Lopes Barbosa ${ }^{1}$, Thays de Oliveira Bezerra ${ }^{2}$, Nardelli Brenda Soares Barros ${ }^{3}$, Claudiane da Silva Lemos ${ }^{4}$, Viviane Nóbrega Gularte Azevedo ${ }^{5}$, Ticiany Alves Bastos ${ }^{6}$, Marcelo Lopes Barbosa ${ }^{7}$, Paulo César de Almeida ${ }^{8}$

Como citar esse artigo: Barbosa AL, Bezerra TO, Barros NBS, Lemos CS, Azevedo VNG, Bastos TA, Barbosa ML, Almeida PC. Caracterização de mães e recém-nascidos pré-termo em uma unidade de terapia intensiva neonatal. Rev Enferm Atenção Saúde [Online]. 2021 [acesso em: ];10(1):e202101. doi: https://doi.org/ 10.18554/reas.v10i1.4660

\section{RESUMO}

Objetivo: Objetivou-se caracterizar o perfil sociodemográfico e clínico dos recém-nascidos pré-termo de uma Unidade de Terapia Intensiva Neonatal. Método: Trata-se de um estudo observacional, descritivo e quantitativo realizado em uma UTIN em Fortaleza-CE, de janeiro a novembro de 2017. A amostra foi composta por 70 recém-nascidos pré-termo. A coleta de dados foi feita por meio de um formulário, contendo as características demográficas e clínicas das mães e dos recém-nascidos. Resultados: Dentre os principais resultados, destacam-se: 69,6\% das mães tinham idade entre 20-34 anos, 22,1\% fizeram cinco consultas de pré-natal, 29,4\% apresentaram doença hipertensiva específica da gestação. Concernente aos recémnascidos, quanto à idade gestacional, a maioria era pré-termo extremo $(85,3 \%)$, extremamente baixo peso $(50 \%)$ e estavam sob ventilação mecânica $(78,6 \%)$. Conclusão: Por meio do

\footnotetext{
${ }^{1}$ Doutora em Cuidados Clínicos em Enfermagem e Saúde. Professora substituta da UFC e Centro Unichristus Fortaleza-CE, Fortaleza-CE. Brasil. andrea_lopes_barbosa@ @otmail.com. ORCID: http://orcid.org/0000-00016132-3987

${ }^{2}$ Graduada pelo Centro Universitário Unigrande. Residente em Terapia Intensiva pela UFC - Fortaleza-CE. Fortaleza CE. ORCID: http://orcid.org/0000-0003-2790-6585

${ }^{3}$ Graduada pelo Centro Universitário Estácio do Ceará. Enfermeira do Hospital Nossa Senhora da Conceição. Especialista em Enfermagem Pediátrica e Neonatal - Fortaleza-CE. Fortaleza CE. ORCID: http://orcid.org/00000001-7200-6274

${ }^{4}$ Graduada pelo Centro Universitário Estácio do Ceará. Enfermeira do Hospital Unimed Fortaleza (Home Care/Pediatria). Especialização em andamento em Nefrologia pelo Centro Universitário Unichristus. - FortalezaCE. Fortaleza CE. ORCID: http://orcid.org/0000-0002-1654-1712

${ }^{5}$ Graduada pelo Centro Universitário Estácio do Ceará. Professora visitante na Escola de Saúde Pública do Ceará. Especialista em Nefrologia - Unichristus, Fortaleza-CE. Mestranda em Enfermagem pela Universidade da Integração Internacional da Lusofonia Afro-Brasileira. Fortaleza-CE. ORCID: http://orcid.org/0000-0002-01340694

6 Graduada pelo Centro Universitário Estácio do Ceará - Fortaleza-CE. Fortaleza-CE. ORCID: http://orcid.org/0000-0002-4479-1989

${ }^{7}$ Médico pela Universidade de Brasília e Universidade Federal do Ceará -Fortaleza-CE. Plantonista da uti da meac da UFC e do Hospital Geral de Fortaleza - Fortaleza-CE. Mestrado em Ciências Médicas pela Universidade de Fortaleza-CE. Fortaleza-CE. ORCID: http://orcid.org/0000-0001-5872-8078

${ }^{8}$ Estatística pela UFC. Doutorado em Saúde Pública - Faculdade de Saúde Pública da USP - São Paulo-SP. Professor Adj. Na UEC, Programa de Pós-graduação em Enfermagem de Cuidados Clínicos em Saúde e Mestrado em Nutrição e Saúde. Professor, colaborador da pós-graduação de Enfermagem da UFC - Fortaleza-CE.FortalezaCE. ORCID: http://orcid.org/0000-0002-2867-802X
} 
conhecimento das características dos recém-nascidos internados, deve-se visar à melhor assistência pré-natal, prever recursos e treinar os profissionais, no intuito de melhorar a assistência à saúde na Unidade de Terapia Intensiva Neonatal.

Descritores: Perfil de Saúde; Mães; Recém-Nascido Prematuro; Unidade de Terapia Intensiva Neonatal.

\begin{abstract}
Objective: The objective was to characterize the sociodemographic and clinical profile of preterm newborns in a Neonatal Intensive Care Unit. Method: This is an observational, descriptive and quantitative study performed in a Neonatal Intensive Care Unit in FortalezaCE, from January to November 2017. The sample was composed of 70 preterm newborns. Data collection took place using a form, containing the demographic and clinical characteristics of mothers and newborns. Results: Among the main results, one highlights: $69.6 \%$ of the mothers were aged between 20 and 34 years, $22.1 \%$ underwent five prenatal consultations, $29.4 \%$ presented hypertensive diseases specific to pregnancy. With regard to newborns, as for gestational age, most were extremely preterm (85.3\%), extremely underweight (50\%) and were undergoing mechanical ventilation $(78.6 \%)$. Conclusion: Through knowledge of the characteristics of hospitalized newborns, we should aim at achieving better prenatal care, providing resources and training professionals, in order to enhance health care in the Neonatal Intensive Care Unit.
\end{abstract}

Descriptors: Health Profile; Mothers; Infant, Premature; Intensive Care Unit, Neonatal.

\title{
RESUMEN
}

Objetivo: El objetivo fue caracterizar el perfil sociodemográfico y clínico de los recién nacidos prematuros en una Unidad de Cuidado Intensivo Neonatal. Método: Estudio observacional, descriptivo y cuantitativo llevado a cabo en una Unidad de Cuidado Intensivo Neonatal en Fortaleza-CE, de Ene. hasta Nov. 2017. La muestra se compuso de 70 recién nacidos prematuros. La recolección de datos tuvo lugar mediante un formulario que contiene las características demográficas y clínicas de las madres y los recién nacidos. Resultados: Entre los principales resultados, se señalan: el 69,6\% de las madres tenían entre 20 y 34 años, el $22,1 \%$ hicieron cinco consultas prenatales, el 29,4\% presentaron una enfermedad hipertensiva específica del embarazo. Con respecto a los recién nacidos, acerca de la edad gestacional, la mayoría eran extremadamente pretérminos $(85,3 \%)$, con un peso extremadamente bajo al nacer $(50 \%)$ y estaban bajo ventilacion mecanica $(78,6 \%)$. Conclusión: A través del conocimiento de las características de los recién nacidos hospitalizados, se debe buscar una mejor atención prenatal, predecir recursos y capacitar a profesionales, con miras a mejorar la atención de salud en la Unidad de Cuidado Intensivo Neonatal.

Descriptores: Perfil de Salud; Madres; Recien Nacido Prematuro; Unidad de Cuidado Intensivo Neonatal.

\section{INTRODUÇÃO}

A prematuridade é um sério problema de saúde pública no mundo, visto ser um dos fatores de risco da morbimortalidade neonatal, influenciando na clínica do recém-nascido $(\mathrm{RN})$ e causando impacto na sua qualidade de vida. $^{1}$

Há uma grande diferença na sobrevida de recém-nascidos pré-termo (RNPTs) dependendo de onde eles nascem. Nos países de alta renda, metade dos RNs com 24 semanas sobrevivem, enquanto que 
nos países de baixa renda metade dessa população com 32 semanas ainda morre devido à falta de cuidados essenciais ao $\mathrm{RN}^{2}$

A taxa de RNPTs tem aumentado mundialmente, chegando a 10,6\% em 2014. O nascimento prematuro no Norte da África está em torno de $13,4 \%$, enquanto na Europa é de $8,7 \% .{ }^{3} \mathrm{O}$ Brasil está entre os dez países com os maiores números de RNPTs e são responsáveis por $60 \%$ dos nascimentos prematuros do mundo. ${ }^{4}$

A prematuridade pode ser $\mathrm{o}$ resultado de uma combinação complexa de condições clínicas, biológicas, genéticas, psicossociais e ambientais, ou mediante recomendação médica devido a fatores de risco maternos e fetais. Fatores maternos como hipertensão, infecções, diabetes, história prévia de parto prematuro, primiparidade e malformação fetal são algumas das causas de prematuridade. ${ }^{5}$

Um estudo transversal realizado com dados de 9.987 registros da base do Sistema de Informações sobre Nascidos Vivos (SINASC) mostrou que extremos de idade materna, tipo de parto e pré-natal com número de consultas insuficientes são também fatores de risco para prematuridade. ${ }^{6}$ Outro estudo evidenciou que o estado civil, a baixa escolaridade e a etnia poderiam influenciar em nascimentos prematuros. $^{7}$
Nesse cenário de tantos fatores que levam à prematuridade, a admissão de um RNPT na Unidade de Terapia Intensiva Neonatal (UTIN) é um momento fundamental para a manutenção do seu quadro clínico e influencia diretamente em sua sobrevida. A prematuridade aumenta o risco de adaptação à vida extrauterina, decorrente, sobretudo, da imaturidade anatomofisiológica.

Os RNPT são, em sua maioria, de alto risco e enfrentam várias desvantagens fisiológicas, como a imaturidade pulmonar, podendo levar à apneia e bradicardia, imaturidade de processos metabólicos, estruturas cerebrais imaturas, maior susceptibilidade a infecções e outras. ${ }^{8}$

Nesse âmbito, é de fundamental importância que os profissionais de saúde, em especial a equipe de enfermagem, tenham conhecimento sobre tudo que envolve o RNPT, para que eles estejam preparados para receber esses pacientes. Esse fato pode favorecer a implementação de melhores estratégias para um cuidado integral e de qualidade. Dessa maneira, este estudo objetivou caracterizar o perfil sociodemográfico e clínico dos RNPTs de uma UTIN.

\section{MÉTODOS}

Trata-se de um estudo observacional, descritivo, de abordagem quantitativa, realizado em uma UTIN de 
referência em pesquisa e gestação de alto risco na cidade de Fortaleza-CE, de janeiro a novembro de 2017.

Foram incluídos na amostra todos os RNPTs internados em leitos de alto risco e que estivessem sob o uso de oxigenoterapia. Para a identificação dos RNs que atendiam a esses critérios, foi realizado um levantamento dos registros de egressos da UTIN, excluindo-se os RNs a termo. A amostra, composta por 70 RNPTs, foi por conveniência.

A coleta de dados foi feita por meio de um formulário, contendo as características demográficas e clínicas das mães dos RNs (idade, procedência, estado civil, escolaridade, ocupação, número de consultas do pré-natal, problemas durante a gestação e parto) e características de base dos recém-nascidos quanto às condições de nascimento (sexo, tipo de parto, peso ao nascer, Apgar, idade gestacional, modalidade de oxigenoterapia instalada ao ser admitido na UTIN). O preenchimento do formulário foi baseado em informações contidas nos prontuários do RNs.

Os resultados foram organizados em tabelas, com as frequências absolutas e percentuais. Calcularam-se as médias e os desvios padrão das variáveis idade, paridade, número de consultas de pré-natal das mães e Apgar, idade gestacional ao nascer e peso dos RNs. Os dados foram processados no SPSS 20.0, licença número 10101131007.

O estudo foi encaminhado ao Comitê de Ética em Pesquisa da instituição na qual foi realizado, sendo aprovado pelo protocolo $\quad \mathrm{n}^{\mathrm{o}} \quad 1.869 .556, \quad \mathrm{CAAE}$ 62667516.7.0000.5050.

\section{RESULTADOS}

Os resultados apresentaram o perfil sociodemográfico e clínico das mães e seus respectivos RNs.

A Tabela 1 apresenta as características sociodemográficas e clínicas das mães durante a gestação e nascimento dos RNs.

Tabela 1 - Distribuição do número de mães segundo características sociodemográficas e clínicas que tiveram seus recém-nascidos admitidos na UTIN, Fortaleza, CE, jan./nov. 2017

\begin{tabular}{|c|c|c|c|c|}
\hline Características & $\mathbf{N}$ & $\%$ & Média & DP \\
\hline Idade & & & 27,3 & $\pm 7,5$ \\
\hline Até 19 anos & 7 & 10,1 & & \\
\hline $20-34$ anos & 48 & 69,6 & & \\
\hline 35 ou mais & 14 & 20,3 & & \\
\hline Não informado & 1 & 1,4 & & \\
\hline Procedência & & & & \\
\hline Fortaleza & 25 & 35,7 & & \\
\hline
\end{tabular}




\section{Estado civil}

Solteira

$17 \quad 25,4$

Casada/ União estável

50

Não informado

$3 \quad 4,3$

Escolaridade

Fundamental

Médio

$29 \quad 41,4$

Superior Incompleto

$34-48,6$

Superior Completo

$4 \quad 5,7$

\begin{tabular}{l} 
Ocupação \\
Dona de Casa \\
Agricultora \\
Costureira \\
Outros \\
Não informado \\
\hline
\end{tabular}

$25 \quad 36,8$

$9 \quad 13,2$

$10 \quad 14,7$

$24 \quad 35,3$

$2 \quad 2,9$

\section{Paridade}

Nulípara

Primípara

Multípara

$\begin{array}{cc}5 & 7,1 \\ 36 & 51,4 \\ 29 & 41,5\end{array}$

$1,6 \quad \pm 1,1$

\begin{tabular}{lccc}
\hline $\mathbf{N}^{\mathbf{0}}$ de consultas de pré-natal & & 4,3 & $\pm 1,8$ \\
0 & 1 & 1,5 & \\
1 & 5 & 7,4 & \\
2 & 8 & 11,8 & \\
3 & 7 & 10,3 & \\
4 & 13 & 19,1 & \\
5 & 15 & 22,1 \\
6 & 8 & 11,8 \\
Mais de 6 & 11 & 16,2 & \\
Não informado & 2 & 2,9 \\
\hline
\end{tabular}

Problemas durante a gestação

\begin{tabular}{lcc} 
DHEG ou Pré-eclâmpsia & 20 & 29,4 \\
ITU & 13 & 19,1 \\
PEG & 3 & 4,4 \\
TPP & 4 & 5,9 \\
Placenta prévia & 1 & 1,5 \\
Vulvovaginites & 2 & 2,9 \\
Outros & 41 & 60,3 \\
Não informado & 16 & 22,8 \\
\hline
\end{tabular}

Tipo de parto

$\begin{array}{lll}\text { Cesárea } & 43 & 61,4\end{array}$

Vaginal

27

38,6

Fonte: prontuários de RNPTs internados em leitos de uma UTIN de uma instituição pública situada em FortalezaCE, jan./nov. 2017.

No tocante às características maternas (Tabela 1), os resultados revelaram que prevaleceu a idade entre 20 34 anos $(69,6 \%)$, com média de 27,3 $\pm 7,5$, sendo a maioria delas provenientes da região metropolitana de Fortaleza $(64,3 \%)$ e casadas/união estável (74,6\%). Em relação à escolaridade, ocupação e paridade, a 
maior parte das mães cursaram o ensino médio $(48,6 \%)$, eram donas de casa $(36,8 \%)$ e primíparas $(51,4 \%)$ com média de $1,6 \pm 1,1$, respectivamente.

Quanto ao pré-natal, apenas 16,2\% das mães fizeram sete ou mais consultas (média de 4,3 $\pm 1,8$ ). Os principais problemas apresentados durante a gestação foram doença hipertensiva específica da gestação - DHEG ou pré-eclâmpsia $(29,4 \%)$ e infecção do trato urinário - ITU $(19,1 \%)$. Encontrou-se que a maioria das mães $(61,4 \%)$ teve seus filhos por parto cesárea. Tabela 2 apresenta as características sociodemográficas e clínicas dos RNs ao nascerem, e logo ao serem admitidos na UTIN.

Tabela 2 - Distribuição do número de recém-nascidos admitidos na UTIN segundo características sociodemográficas e clínicas, Fortaleza, CE, jan./nov. 2017

\begin{tabular}{|c|c|c|c|c|}
\hline Características & $\mathbf{N}$ & $\%$ & Média & DP \\
\hline \multicolumn{5}{|l|}{ Sexo } \\
\hline Masculino & 35 & 50,0 & & \\
\hline Feminino & 35 & 50,0 & & \\
\hline \multicolumn{5}{|l|}{ Tipo de parto } \\
\hline Cesárea & 43 & 61,4 & & \\
\hline Vaginal & 27 & 38,6 & & \\
\hline Apgar $1^{\circ}$ min & & & 5,4 & $\pm 1,9$ \\
\hline Até 6 & 41 & 60,3 & & \\
\hline $7-10$ & 27 & 39,7 & & \\
\hline Não informado & 2 & 2,9 & & \\
\hline Apgar $5^{\circ} \min$ & & & 7,5 & $\pm 1,5$ \\
\hline Até 6 & 12 & 17,9 & & \\
\hline $7-10$ & 55 & 82,1 & & \\
\hline Não informado & 3 & 4,3 & & \\
\hline Idade gestacional ao nascer (sem) & & & 27,9 & $\pm 2,4$ \\
\hline Pré-termo Extremo $(\leq 30)$ & 58 & 85,3 & & \\
\hline Pré-termo Moderado (31 - 34) & 9 & 13,2 & & \\
\hline Pré-termo Limítrofe (35 - 36) & 1 & 1,5 & & \\
\hline Não informado & 2 & 2,9 & & \\
\hline Peso ao nascer (g) & & & 1070,4 & $\pm 392,7$ \\
\hline Extremamente Baixo Peso $(<1000)$ & 35 & 50,0 & & \\
\hline Muito Baixo Peso (1000-1499) & 27 & 38,6 & & \\
\hline Baixo Peso (1500-2499) & 8 & 11,4 & & \\
\hline \multicolumn{5}{|c|}{ Modalidade de oxigenoterapia ao ser admitido na } \\
\hline VM & 55 & 78,6 & & \\
\hline VNI & 6 & 8,6 & & \\
\hline Cpap Nasal & 9 & 12,9 & & \\
\hline
\end{tabular}


No que concerne ao sexo dos RNPTs, houve igual distribuição percentual, a maioria $(61,4 \%)$ nasceu de parto cesárea, o Apgar que prevaleceu no $1^{\circ}$ minuto $(60,3 \%)$ foi até 6 com uma média de $5,4 \pm 1,9$ e, no $5^{\circ}$ minuto, de 7 a $10(82,1 \%)$,

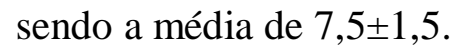

Quanto à idade gestacional dos RNs, peso ao nascer e modalidade de oxigenoterapia utilizada ao serem admitidos na UTIN, os resultados foram que o maior número de RNs era pré-termo extremo $(85,3 \%)$ com uma média de 27,9 $\pm 2,4$ semanas, extremamente baixo peso (50\%), sendo a média de $1070,4 \pm 392,7 \mathrm{~g}$, e estava sob ventilação mecânica $(\mathrm{VM})(78,6 \%)$, respectivamente (Tabela 2 ).

\section{DISCUSSÃO}

Neste estudo, constatou-se a predominância de mães com idade entre 2034 anos, provenientes da região metropolitana de Fortaleza, casadas/união estável, com ensino médio e donas de casa, corroborando com um estudo realizado em uma UTIN do Distrito Federal. ${ }^{9}$

Ainda assim, extremos de idade materna é um dos atores que influenciam no nascimento de RNPTs. ${ }^{10} \mathrm{O}$ presente estudo foi de encontro com essa afirmação, visto que a maior parte das mães dos RNs encontravam-se na faixa etária adulta- jovem. Isso pode estar relacionado a um pré-natal incompleto.

A regionalização é uma das diretrizes do Sistema Único de Saúde e orienta a descentralização das ações e serviços de saúde, possibilitando, assim, melhor assistência obstétrica, de modo a atender às necessidades das gestantes em um determinado território. ${ }^{11}$ Dessa maneira, observa-se que os resultados desse estudo corroboram com a ideia, pois a maior parte das mães internadas era proveniente da região metropolitana de Fortaleza.

Em relação ao estado civil, a maioria das mães dos RNPTs eram casadas/união estável. Destaca-se esse fato para que seja inclusa a paternidade em todo o ciclo gravídico puerperal, em vista dos benefícios que a participação do homem traz quando se mostra afetivo, aumentando a segurança da mulher e o vínculo familiar consistente, do contrário, uma desagregação familiar pode levar a um trabalho de parto prematuro. $^{12}$

A escolaridade materna tem sido apresentada como variável independente em trabalhos epidemiológicos de diferentes temáticas. Assim, uma baixa escolaridade materna está associada a um risco maior de mortalidade materna e neonatal. ${ }^{13}$

A relação entre ocupação e o trabalho de parto prematuro entre as mães estudadas revelou que a maior parte 
realizava alguma atividade. A função de dona de casa, que se verificou na maioria das mulheres, de costureira e de agricultora se configuram como empregos que exigem esforço físico, permanecendo a gestante por longo período na posição ortostática ou sentada. Essas características vêm sendo vinculadas à prevalência de partos prematuros. $^{14}$

No que se refere às características clínicas das mães, a maioria era primípara e realizou entre quatro e cinco consultas de pré-natal. Os problemas que ocorreram com maior frequência durante a gestação nessa população foram pré-eclâmpsia, seguida de infecção do trato urinário. O tipo de parto de maior frequência foi a cesárea.

Um estudo caso-controle realizado com 111 puérperas de uma instituição pública da Bahia identificou os fatores maternos mais associados ao nascimento de RNs com baixo peso e prematuros. A maior parte das puérperas tinham menos de seis consultas de pré-natal, tiveram préeclâmpsia e infecção de trato urinário como principais complicações no período gestacional, sendo a maioria multípara, optando por parto vaginal. Os resultados demonstraram que as puérperas expostas ao pré-natal inadequado apresentaram mais chance de terem $\mathrm{RN}$ com baixo peso e parto prematuro. ${ }^{15}$ Tal pesquisa está de acordo com o presente estudo quanto ao número inadequado de consultas de pré-natal e os problemas apresentados durante a gestação, porém as mães deste estudo eram primíparas.

Em relação às características dos RNPTs admitidos na UTIN, os resultados evidenciaram uma uniformidade quanto ao sexo e a maior parte nasceu de parto cesárea. Esse parto, em gestações de alto risco, tem sido considerado um procedimento importante para a redução dos agravos perinatais, elevando a sobrevida dos RNs, por exemplo, em casos de sofrimento fetal, falta de progresso no trabalho de parto vaginal, apresentação pélvica, pré-eclâmpsia, dentre outros. ${ }^{16}$

Logo após o nascimento, ocorrem profundas mudanças fisiológicas no $\mathrm{RN}$, pois ele necessita de adaptação à vida extrauterina, precisando de avaliação, sendo o boletim de Apgar utilizado para mensurar sua vitalidade. Neste estudo, uma grande parte dos RNs apresentaram um Apgar baixo no primeiro minuto, no entanto, no quinto minuto, houve uma melhora da vitalidade dos RNs e a maioria obteve um Apgar de 7-10. Essa melhora significativa dos RNs, comparando o primeiro com o quinto minuto, está relacionada a uma boa conduta dos profissionais de saúde em sala de parto, tanto no atendimento à mulher quanto ao neonato. Destaca-se que a principal recomendação para diminuir a mortalidade materna e neonatal é o 
atendimento destes por um pessoal qualificado. ${ }^{17}$

Uma pesquisa retrospectiva descritiva realizada em um hospital no interior do Ceará objetivou avaliar a vitalidade de RNs por meio do índice de Apgar, relacionando às características materno-obstétricas. A relação do tipo de parto com a vitalidade do recém-nascido, avaliado com o índice de Apgar, não obteve resultados com diferenças significativas, pois os RNs obtiveram um resultado satisfatório para os dois tipos de parto, cesárea e vaginal. Tornou-se claro que a vitalidade do $\mathrm{RN}$ está diretamente relacionada a fatores como idade gestacional, faixa etária materna e consultas de pré-natal. ${ }^{18}$ Essa pesquisa confirma os dados do presente estudo, pois todos os RNs eram pré-termo e o número de consultas de pré-natal foi insuficiente.

No que se refere à idade gestacional, os RNPTs podem ser subclassificados em: pré-termo limítrofe (35 a 36 semanas de IG), pré-termo moderado (31 a 34 semanas de IG) e prétermo extremo ( $\mathrm{IG} \leq 30$ semanas). Em relação ao peso, os RNs devem ser considerados de baixo peso ao nascer (menos do que $2.500 \mathrm{~g}$ ), muito baixo peso (menos do que $1.500 \mathrm{~g}$ ) e extremo baixo peso (menos do que $1.000 \mathrm{~g}$ ). ${ }^{19}$

Quanto à idade gestacional e ao peso ao nascer, o que predominou neste estudo foram os RNPT extremos, seguidos da prematuridade moderada e limítrofe; extremo baixo peso, seguidos dos de muito baixo peso e baixo peso. A modalidade de oxigenoterapia mais presente em ambos os grupos foi a VM.

Um estudo retrospectivo e documental de um setor Canguru que admitiu RNPTs provenientes da UTIN de um hospital de referência em Fortaleza-CE pesquisou o perfil desses pacientes. Houve evidência de que a maioria nasceu de parto cesárea (76,6\%), com Apgar $\geq 7(63,3 \%)$ no primeiro minuto e Apgar $\geq 7(96,6 \% \%)$ no quinto minuto. Quanto à idade gestacional, a maioria $(56,6 \%)$ era de pré-termo extremos, seguidos de $36,6 \%$ de pré-termo moderados e 6,6\% de limítrofes. Quanto ao peso, $56,6 \%$ eram de extremo baixo peso, $40 \%$ muito baixo peso e $3,3 \%$ baixo peso, sendo que $100 \%$ tiveram síndrome do desconforto respiratório como diagnóstico de admissão na UTIN e, destes, 53,3\% estiveram em uso de VM, 83,3\% usaram Cpap Nasal e 90,0\% Oxi-Hood..$^{20}$

Esses resultados estão de acordo com o presente estudo, visto que a maioria dos partos foi cesárea, a maior parte dos neonatos eram pré-termo extremos, com extremo baixo peso e estiveram em uso de VM. Todavia, o estudo em questão foi de encontro com a pesquisa supracitada apenas no que se refere ao boletim de Apgar no primeiro minuto. Isso pode estar associado 
a uma maior hipóxia sofrida pelos pacientes ao nascer quando se trata do estudo em questão. Ademais, a maior parte dos RNs eram pré-termo extremos e com extremo baixo peso.

\section{CONCLUSÃO}

Este estudo constatou as principais características sociodemográficas e clínicas das mães identificadas, sendo a maioria mães adultas jovens, provenientes da região metropolitana de Fortaleza, com ensino médio, donas de casa, casadas/união estável e primíparas. Dessa maneira, pôde-se perceber que, apesar dessas mães apresentarem características favoráveis ao desenvolvimento de um concepto saudável, exceto pelo baixo nível de escolaridade, seus filhos necessitaram de uma assistência mais especializada.

Observou-se também que a média de consultas de pré-natal das mães foi inadequada e que a maior parte delas enfrentou problemas durante a gestação, como DHEG e ITU. Além disso, o parto cesárea foi o mais prevalente. Com isso, constatou-se que há uma grande demanda dessas mulheres da região metropolitana para a capital, evento que poderia ser evitado se alguns problemas durante a gestação fossem detectados precocemente no pré-natal.

Quanto ao perfil dos RNPTs admitidos na UTIN, o presente estudo revelou que, com relação ao sexo, masculino e feminino estiveram em igual proporção. Referente ao Apgar, os RNs conseguiram uma melhora importante do primeiro para o quinto, sendo que o maior número de pacientes eram pré-termo extremos, com extremo baixo peso e estavam sob VM.

Destaca-se que, ao avaliar os resultados deste estudo, percebeu-se que alguns dados maternos dos RNs analisados podem ter contribuído para o agravamento da saúde dos mesmos, uma vez que grande parte de suas genitoras não realizaram um acompanhamento de pré-natal adequado.

Ademais, ressaltam-se os elevados percentuais de prematuridade extrema e de extremo baixo peso de nascimento encontrados, além de outras comorbidades que contribuíram para a admissão na UTIN, as quais poderiam ter sido evitadas ou tratadas precocemente com o auxílio de adequadas intervenções pré-natais e perinatais de menor custo e complexidade. Para mais, devido à idade gestacional e ao peso, um número significante de pacientes estava em uso de VM.

Diante disso, por meio do conhecimento das características dos RNs internados, deve-se visar à melhor assistência pré-natal, prever recursos e treinar os profissionais, com o intuito de melhorar a assistência à saúde na UTIN e, ainda, no período pré-natal. 
Como limitação desse estudo, citase a sua realização em uma única UTIN, portanto sugere-se uma maior abrangência em futuros estudos nesse contexto.

\section{REFERÊNCIAS}

1. Oliveira LL, Gonçalves AC, Costa JSD, Bonilha ALL. Fatores maternos e neonatais relacionados à prematuridade. Rev Esc Enferm USP. [Internet]. 2016 [citado em 8 out 2020]; 50(3):382-89. Disponível em: https://www.scielo.br/pdf/reeusp/v50n3/pt _0080-6234-reeusp-50-03-0382.pdf 2. World Health Organization. Born too soon: the global action report on preterm birth [Internet]. Geneva: WHO; 2012 [citado em 31 maio 2020]. Disponível em: https://www.who.int/pmnch/media/news/2 012/201204_borntoosoon-report.pdf 3. Chawanpaiboon S, Vogel JP, Moller AB, Lumbiganon P, Petzold M, Hogan D, et al. Global, regional, and national estimates of levels of preterm birth in 2014: a systematic review and modelling analysis. Lancet Glob Health [Internet]. 2019 [citado em 08 dez 2020]; 7(1):e3746. Disponível em:

https://pubmed.ncbi.nlm.nih.gov/30389451 /

4. World Health Organization. Preterm birth [Internet]. Geneva: WHO; 2015 [citado em 31 maio 2020]. Disponível em: http://www.who.int/mediacentre/factsh eets/fs363/en/

5. Tamez R. Enfermagem na UTI neonatal: assistência ao recém-nascido de alto risco. 3ed. Rio de Janeiro: Guanabara Koogan; 2017. Gravidez e parto de alto risco. cap. 2, p. 14-16.

6. Guimarães EAA, Vieira CS, Nunes FDD, Januário GC, Oliveira VC, Tibúrcio JD. Prevalência e fatores associados à prematuridade em Divinópolis, Minas Gerais, 2008-2011: análise do Sistema de Informações sobre Nascidos Vivos.
Epidemiol Serv Saúde. 2017; 26(1):91-98.

Disponível em:

https://www.scielo.br/pdf/ress/v26n1/2237 -9622-ress-26-01-00091.pdf

7. Sadovsky ADI, Matijasevich A, Santos IS, Barros FC, Miranda AE, Silveira MF. Iniquidades socioeconômicas em nascimentos prematuros em quatro estudos brasileiros de coortes de nascimento. J Pediatr. (Rio J.). 2018; 94(1):15-22.

Disponível em: https://www.scielo.br/pdf/jped/v94n1/pt_0 021-7557-jped-94-01-0015.pdf

8. Rosenberg AA, Grover T. O recémnascido. In: Hay Jr. WW, Deterding RR, Levin MJ, Abzug MJ. Current: diagnóstico e tratamento. Porto Alegre: AMGH; 2016. cap. 2, p. 9-74.

9. Ferraresi MF, Arrais AR. Perfil epidemiológico de mães de recém-nascidos admitidos em uma unidade neonatal pública. Rev Rene. 2016; 17(6):733-40.

Disponível em:

http://www.periodicos.ufc.br/rene/article/vi ew/6453/4702

10. Ministério da Saúde (Brasil), Secretaria de Atenção à Saúde. Atenção ao pré-natal de baixo risco [Internet]. Brasília, DF: Ministério da Saúde; 2012 [citado em 31 maio 2020]. (Cadernos de Atenção Básica; n.32). Disponível em:

http://bvsms.saude.gov.br/bvs/publicacoes/ cadernos_atencao_basica_32_prenatal.pdf 11. Gryschek ALFPL, Nichiata LYI, Fracolli LA, Oliveira MAF, Pinho PH. Tecendo a rede de atenção à saúde da mulher em direção à construção da linha de cuidado da gestante e puérpera, no Colegiado de Gestão Regional do Alto Capivari. Saúde Soc. 2014; 23(2):689-700. Disponível em:

https://www.scielo.br/pdf/sausoc/v23n2/01 04-1290-sausoc-23-2-0689.pdf

12. Petito ADC, Cândido ACF, Ribeiro

LO, Petito G. A importância da participação do pai no ciclo gravídico puerperal: uma revisão bibliográfica. Revista Eletrônica da Faculdade de Ceres [Internet]. 2015 [citado em 31 maio 2020]; 4(1): 1-14. Disponível em: 
http://periodicos.unievangelica.edu.br/inde x.php/refacer/article/view/3367

13. Gonzaga ICA, Santos SLD, Silva ARV, Campelo V. Atenção pré-natal e fatores de risco associados à prematuridade e baixo peso ao nascer em capital do nordeste brasileiro. Ciênc Saúde Colet. 2016; 21(6):1965-74. Disponível em: https://www.scielo.br/pdf/csc/v21n6/14138123-csc-21-06-1965.pdf 14. Montenegro CAB, Rezende Filho J. Obstetrícia fundamental. 13ed. Rio de Janeiro: Guanabara Koogan; 2014. 15. Carvalho SS, Coelho JMF, Bacelar DÂ, Mariola E. Fatores maternos para o nascimento de recém-nascidos com baixo peso e prematuros: estudo caso-controle. Ciênc Saúde (Porto Alegre). 2016; 9(2):7682. Disponível em:

file://C:/Users/andre/Downloads/21947-

Texto\%20do\%20artigo-102769-2-1020160916\%20(1).pdf

16. Silva CF, Leite ÁJM, Almeida NMGS, Leon ACMP, Olofin I, Castro ECM et al. Fatores associados ao óbito neonatal de recém-nascidos de alto risco: estudo multicêntrico em Unidades Neonatais de alto risco no nordeste brasileiro. Cad Saúde Pública [Internet]. 2014 [citado em 31 maio 2020]; 30(2):355-68. Disponível em: http://www.scielo.br/pdf/csp/v30n2/0102311X-csp-30-2-0355.pdf

17. Esser MAMS, Mamede FV, Mamede MV. Perfil dos profissionais de enfermagem que atuam em maternidades em Londrina, PR. Rev Eletrônica Enferm. [Internet]. 2012 [citado em 31 maio 2020]; 14(1):133-41. Disponível em: https://www.revistas.ufg.br/fen/article/vie w/11032/15565

18. Muniz EB, Vasconcelos BB, Pereira NA, Frota RG, Moraes CEB, Oliveira MAS. Análise do boletim de Apgar em dados do Sistema de Informação sobre Nascidos Vivos registrados em um hospital do interior do estado do Ceará, Brasil. Revista de Medicina e Saúde de Brasília [Internet]. 2016 [citado em 31 maio 2020]; 5(2):182-91. Disponível em: https://portalrevistas.ucb.br/index.php/rms br/article/view/6677/4563

19. Leone CR, Ramos JL, Vaz FA. O recém nascido pré-termo. In: Marcondes E, Vaz FA, Ramos JL, Okay Y. Pediatria básica. 9th ed. São Paulo: Savier; 2002. p. 348-352. Cap 8

20. Souza KCL, Carvalho ACF, Evangelista NMC, Nascimento MM, Braide ASG, Silveira MGAO. Profile of newborns discharged from the intensive neonatal care unit submitted to the kangaroo ward. J Contemp Pediatr. 2017; 4(3):685-90. Disponível em: file:///C:/Users/andre/Downloads/6343252-1-PB.pdf

RECEBIDO: $13 / 06 / 2020$

APROVADO: 02/11/2020

PUBLICADO: 01/2021 